\title{
Maths 'Nobel' rumoured for Russian recluse
}

Some speculation always precedes the announcement of the Fields medals, the most illustrious awards in mathematics. But this year rumours have an extra dimension: one of the prizes to be awarded at the International Congress of Mathematicians in Madrid on 22 August may be given for the solution of a century-old problem.

Favourite to win a medal is Russian mathematician Grigory 'Grisha' Perelman, for his work on the Poincaré conjecture. Three papers now suggest that his proof of the conjecture is right. If it stands up to two years of scrutiny, Perelman will also be eligible for at least a share of a million-dollar mathematics prize (see 'Clay feats').

"I am completely convinced that Perelman has proved the Poincaré conjecture," says John Morgan, a topologist at Columbia University in New York and co-author of the most
"For people studying topology, the whole landscape has changed."
The geometrization conjecture classifies all the possible forms that three-dimensional surfaces, called three-manifolds, can take. The Poincaré conjecture describes the properties of a subset known as 'simply connected' manifolds - in particular, those for which every loop drawn on the surface can be shrunk to a point.

To tackle the conjecture, Perelman used a mathematical tool known as Ricci flow, developed by Richard Hamilton of Columbia University. Perelman, formerly of the Steklov Institute in Moscow, advanced Hamilton's work by finding a way to deal with certain problems encountered in using Ricci flow to study the deformation of surfaces.

The advent of a possible proof shook a small contingent of mathematicians. "For people studying topology, the whole landscape has changed. It's like waking up one morning after recent paper analysing Perelman's work.

The Poincaré conjecture is a seemingly simple statement about the nature of threedimensional surfaces, but it had resisted proof since French mathematician Henri Poincaré put it forward in 1904.

In 2002, Perelman posted to an online preprint server a paper ${ }^{1}$ that claimed to "give a sketch of an eclectic proof of this conjecture". By this, Perelman meant not just the Poincaré conjecture, but the geometrization conjecture, a much broader theorem of which Poincarés statement is a special case.

an earthquake," says Bruce Kleiner of Yale University in New Haven, Connecticut.

Perelman followed his first article with two further papers, and in the spring of 2003 he toured the United States to lecture about his work $^{2}$. He has since retreated from the public eye, leaving other mathematicians to comb through his papers line by line, filling in details and searching for holes in his logic.

"How do you decide whether anything is correct?" asks John Ball, president of the International Mathematical Union and chair of the Fields medal committee. "All that can happen

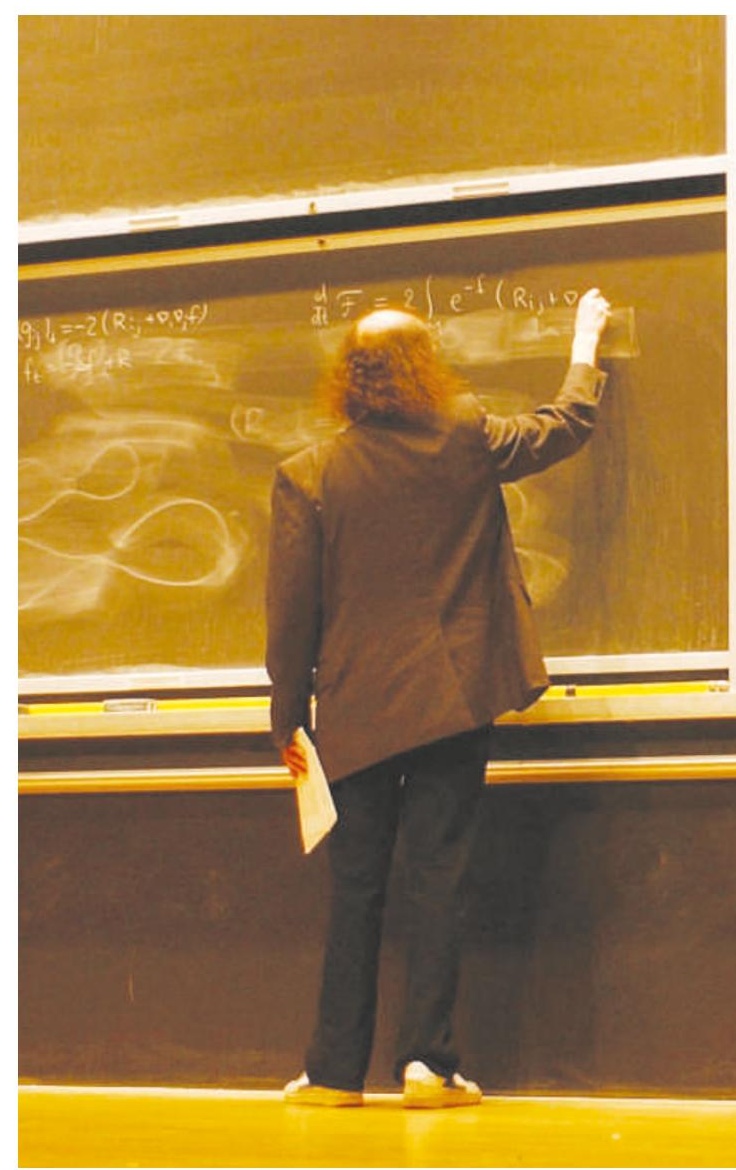

is that clever people, experts in the area, read it and come to an opinion."

Three pairs of respected mathematicians have produced written accounts filling in the details of Perelman's work.

Kleiner and John Lott of the University of Michigan, Ann Arbor, posted their most recent paper to the arXiv preprint server on $25 \mathrm{May}^{3}$. "There was a moment when I really thought there was a problem with the argument," says Kleiner. "But that state of mind

\section{Bird flu not set for pandemic, says US team}

\section{Researchers have tried to create a} pandemic H5N1 influenza strain and failed.

Simply mixing genes from an H5N1 bird-flu strain with those from an H3N2 human strain did not result in a strain that was readily transmissible, at least among ferrets. The scientists who conducted the work, at the Centers for Disease Control and Prevention (CDC) in Atlanta, Georgia, say it suggests that the
H5N1 virus will require a complex series of genetic changes to evolve into a pandemic strain.

"These data do not mean that H5N1 cannot convert to become transmissible from person to person," says Julie Gerberding, director of the CDC. "We are not out of the woods on pandemic preparedness yet."

Others agree, pointing out that there are many ways a pandemic strain could evolve. For instance, strains other than those used in the experiments could get together.

"They need to look at other viruses, because both human and avian flu continue to evolve," says Frederick Hayden, a flu specialist at the University of Virginia, Charlottesville, who is currently working with the World Health Organization.

Since 1997, millions of domestic and wild birds have died owing to the H5N1 strain of flu. It has infected at least 232 people and killed 134 of them. Scientists are worried that H5N1 will learn to pass easily between humans and kill millions more. In 1957 and 1968, pandemic strains of flu seem to have emerged when bird and human flu viruses exchanged genes, allowing the birdflu virus to be easily transmitted between people. 

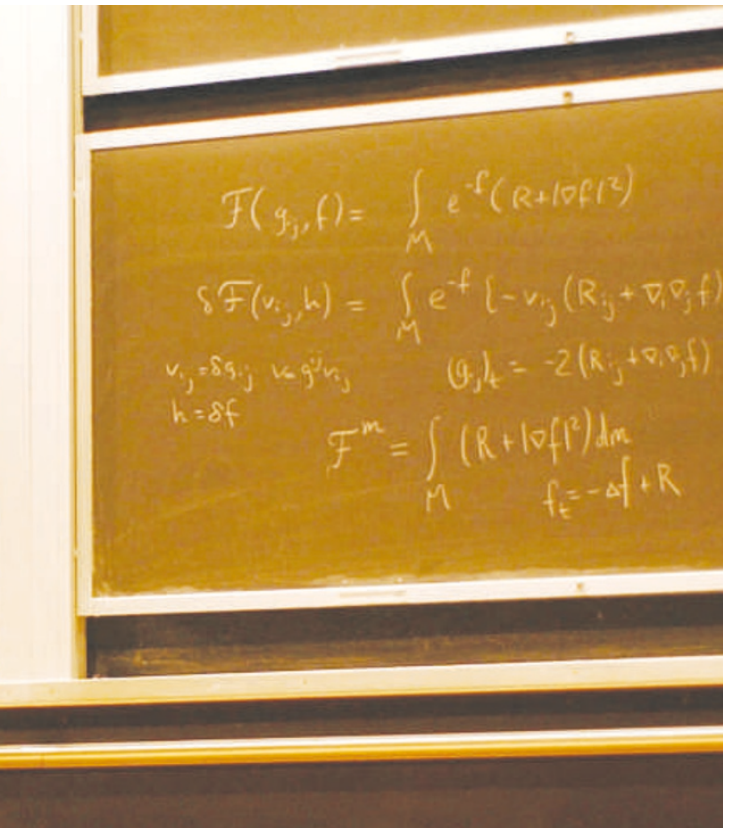

Board meeting: Grigory Perelman presents his proof of the intractable Poincaré conjecture.

persisted for only a week or two before the issue was resolved."

A 473-page paper ${ }^{4}$ by Morgan and Gang Tian of the Massachusetts Institute of Technology appeared on 25 July.

The third paper, from Huai-Dong Cao of Lehigh University in Bethlehem, Pennsylvania, and Xi-Ping Zhu of Zhongshan University

\section{Clay feats: the million-dollar questions}

In 2000, the privately funded Clay Mathematics Institute in Cambridge, Massachusetts, announced a prize of $\$ 1$ million for the solution of each of seven mathematical conundrums - including the Poincaré conjecture.

"I didn't think that I would live to see the Poincaré conjecture solved," says James Carlson, president of the institute. "But we're there, or almost there."

The rules require that the proof appear in a peerreviewed outlet and then survive two years of scrutiny. Grigory Perelman's papers containing a possible proof of the Poincaré conjecture

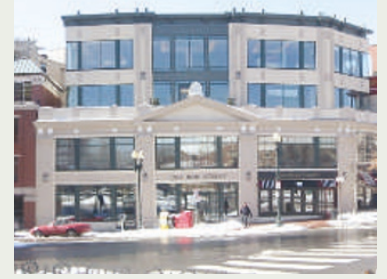

Great foundations: the Clay Mathematics Institute.

appear online but have not been formally reviewed. Those who have tried to persuade Perelman to publish say that he shows no interest. Other recent papers on his work by different mathematicians may count instead, says Carlson. "These serve to start the clock on the waiting period," he says.

Some mathematicians suggest that the prize money should be shared between Perelman and Richard Hamilton, a mathematician at Columbia University in New York, who laid much of the groundwork. Those who have followed up the proof could also theoretically lay claim to the money.

Bruce Kleiner of Yale University says he will not seek a share. "I'm not saying that it was easy to fill in the details," he says. "But you can't compare it to the level of ingenuity required to sketch out the proof."

J.H. in Guangzhou, China, was published in June ${ }^{5}$. It claims to complete the proof of both conjectures, rather than simply fleshing out Perelman's work. This is justified, argues Shing-Tung Yau, an editor-in-chief on the journal, because Cao and Zhu follow a somewhat different argument to Perelman.

The appearance of these three papers has fuelled rumours that Perelman will receive a Fields prize. Up to four medals are awarded every four years to mathematicians no older than 40, making Perelman just eligible for this year's crop. Not that he will attend the Madrid meeting: the organizing committee received no reply when they invited him to give a plenary lecture.

Even so, many mathematicians think Perelman will be honoured with a medal. "I hope that they give him one. This is such a major achievement in mathematics, it would be funny if they didn't," says Morgan. There is also a precedent. Two previous Fields medals have been awarded for proofs of an equivalent of the Poincaré conjecture in higher dimensions to Stephen Smale in 1966 and Michael Freedman in 1986.

And in 2006? Morgan, along with 4,000 guests, will find out in a few weeks - when the medals are announced at the opening ceremony of the congress in Madrid.

\section{Jenny Hogan}

1. Perelman, G. Preprint at http://arxiv.org/abs/math.DG/
O211159 (2002).
2. Singer, E. Nature 427, 388-389 (2004).
3. Kleiner, B. \& Lott, J. Preprint at http://arxiv.org/abs/
math.DG/0605667 (2006).
4. Morgan, J. W. \& Tian, G. Preprint at http://arxiv.org/abs/
math.DG/0607607 (2006).
5. Cao, H.-D. \& Zhu, X.-P. Asian J. Math. 10, 165-492 (2006)

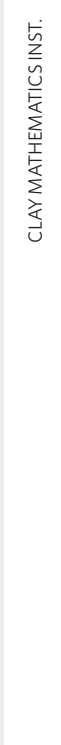

